\title{
Methods of calculation of a friction coefficient: Application to the nanotubes
}

\author{
J. Servantie and P. Gaspard \\ Center for Nonlinear Phenomena and Complex Systems \\ Université Libre de Bruxelles, Code Postal 231, Campus Plaine, 1050 Brussels, Belgium.
}

(Dated: July 27, 2021)

\begin{abstract}
In this work we develop theoretical and numerical methods of calculation of a dynamic friction coefficient. The theoretical method is based on an adiabatic approximation which allows us to express the dynamic friction coefficient in terms of the time integral of the autocorrelation function of the force between both sliding objects. The motion of the objects and the autocorrelation function can be numerically calculated by molecular-dynamics simulations. We have successfully applied these methods to the evaluation of the dynamic friction coefficient of the relative motion of two concentric carbon nanotubes. The dynamic friction coefficient is shown to increase with the temperature.
\end{abstract}

PACS numbers: $61.48 .+\mathrm{c}, 62.20 . \mathrm{Qp}$

Thanks to recent developments in nanotechnology, the hope is high to build mechanical devices on the scale of the nanometer. For this purpose, it is important to determine the mechanical properties and, especially, the friction forces in such nanodevices. In this work, we are going to focus on systems of carbon nanotubes. Our particular interest is for a system that Cumings and Zettl [1] observed experimentally with a TEM. They fixed an edge of a multiwalled nanotube to a surface and opened the other edge, they then extracted inner layers from the core for several nanometers and released them. They observed a full retraction of the inner layers and furthermore, they could conclude that the multiwalled nanotubes are self cleaning since amorphous carbon due to the opening of the edge are inside the tube and also do not have any wear, structural change or fatigue after several extraction and retraction processes. This experiment suggest us that the multiwalled nanotubes can be promising systems for future nanometric mechanical parts such as springs, gears or even motors.

A short time after the work of Cumings and Zettl, Zheng and Jiang [3] estimated the frequency of the oscillations in this system to be of the order of GHz. This result is also very interesting since in the macroscopic world moving parts with such frequencies does not exist at the present time. But as in the macroscopic world moving parts have friction forces which hinder the motion and dissipate energy. We hence have to know the importance of these forces before conceiving such devices. Our work will thus focus on, firstly methods of calculation of the friction and secondly an application of these theories to the multiwalled nanotubes.

The plan of the paper will be as follows, we are first going to introduce the theoretical framework for the description of the mechanics of nanotubes. We will then solve the classical equations of motion and calculate the dynamic friction coefficient by molecular-dynamics simulation and by the autocorrelation-function method developed by Jarzynski, Berry and Robbins [ $[$, 9$]$.

We have depicted in Fig. 1 two sliding nanotubes. $R$ is the distance between the centers of mass of each nan- otube. The Hamiltonian of the system of two nanotubes

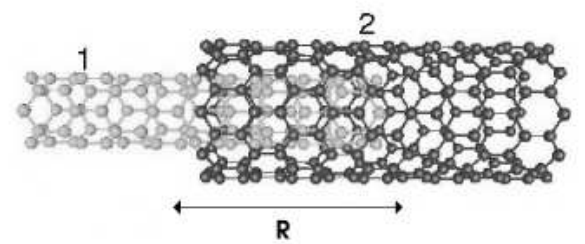

FIG. 1: Sliding nanotubes.

is given by

$$
H=T_{1}+T_{2}+V_{\mathrm{TB}}^{(1)}+V_{\mathrm{TB}}^{(2)}+\frac{1}{2} \sum_{i j} V_{\mathrm{LJ}}\left(\mathbf{r}_{i}^{(1)}-\mathbf{r}_{j}^{(2)}\right)
$$

where $T_{1}$ and $T_{2}$ are respectively the total kinetic energies of the inner and outer tubes, $V_{\mathrm{TB}}^{(1)}$ and $V_{\mathrm{TB}}^{(2)}$ are the total Tersoff-Brenner potentials of both tubes. We use the set of parameters which optimizes the geometric structure [2]. The last term is the interaction potential between the tubes, which is taken as a 6-12 Lennard-Jones potential calculated by Lu and Yang [4] and widely used for molecular-dynamics simulations of nanotubes $3,3,4,[5,6]$.

The calculation of the friction coefficient will be done by using an adiabatic approximation since there are two different time scales in the system. The fast time scale is the one of the vibrations of the atoms which is of the order of the $f s$ and the slow time scale is the one of the motion of the mass centers of the tubes which is of the order of the $p s$ in our case. Thanks to this difference in time scales, we can use a method developed by Jarzynski, Berry and Robbins [8, 9] which is based on the fact that the following quantity is an adiabatic invariant 10, 11],

$$
\Omega(E, \epsilon t)=\int d \mathbf{z} \theta[E-H(\mathbf{z}, \epsilon t)]
$$

where $\epsilon$ is a small parameter, E the energy of the fast system and $H$ is an ergodic adiabatic Hamiltonian. This 
means that $H$ slowly evolves with time and that, at any instantaneous time, $H$ produces trajectories which explore their energy shells ergodically and chaotically. $\mathbf{z}=(\mathbf{q}, \mathbf{p})$ represents the fast phase-space variables. The smallness of the parameter $\epsilon$ guarantees that the motion of vibration is fast compared to the motion of the mass centers. The other important assumption is that the force on the slow system can be expressed as an average over the fast degrees of freedom since the time scale of the fast system is much smaller. We hence write the force as

$$
\mathbf{F}=-\int d \mathbf{z} \rho \nabla H
$$

where $\rho$ is the probability density of the fast system and $\nabla=\partial / \partial \mathbf{R}$ is the derivative with respect to the slow variable. In our specific case $\mathbf{R}$ is the distance between the mass centers of the tubes. Using the fact that there are two different time scales, $\rho$ is expanded as

$$
\rho(\mathbf{z}, t)=\rho_{0}(\mathbf{z}, \mathbf{R})+\epsilon \rho_{1}(\mathbf{z}, \mathbf{R}, t)+\cdots
$$

where the first order term $\rho_{0}$ depends only on the slow time which is regarded as the parameter $\mathbf{R}$. The evolution of $\rho$ is given by the Liouville equation. Injecting the expansion (4) in the Liouville equation gives a hierarchy of equations which can be solved using a microcanonical distribution for $\rho_{0}$. Having calculated $\rho$ to the first order in $\epsilon$ one can find the following reaction force,

$$
\mathbf{F}=-\nabla E-\mathbf{\Gamma} \cdot \dot{\mathbf{R}}
$$

where the first order term is the Born-Oppenheimer force with the energy of the fast system $E$ and the second term the friction force where the velocity $\dot{\mathbf{R}}$ of order $\epsilon$ appears. The dynamic friction coefficients $\boldsymbol{\Gamma}$ are given by

$$
\Gamma_{i j}=\frac{1}{\partial_{E} \Omega} \partial_{E}\left(\partial_{E} \Omega \int_{0}^{\infty} d \tau C_{i j}(\tau)\right)
$$

where $\partial_{E}$ is the partial derivative with respect to the energy of the degrees of freedom of vibration $E$ and $\Omega(E, \mathbf{R})$ is defined by Eq. (2). The autocorrelation function is given by the following expression

$$
C_{i j}(\tau)=\left\langle\partial_{i} \tilde{H}_{\tau} \partial_{j} \tilde{H}\right\rangle_{E, \mathbf{R}}
$$

where $\tilde{H}=H(z, \mathbf{R})-E(\mathbf{R})$ represents the fluctuations of the Hamiltonian. We can simplify the expression of the friction in Eq. (6) by using the Boltzmann equation $S=$ $k_{\mathrm{B}} \ln \partial_{E} \Omega$ and the thermodynamic relation $\partial E / \partial S=T$. Moreover, the adiabatic parameter $\mathbf{R}$ is one-dimensional in our system since the relative motion of the tubes is mainly in the longitudinal direction. We hence get

$$
\Gamma=\left(\beta+\partial_{E}\right) \int_{0}^{\infty} d \tau C(\tau) \approx \beta \int_{0}^{\infty} d \tau C(\tau)
$$

with $\beta=1 / k_{\mathrm{B}} T$. In Eq. (8), we have used the result that the second term with $\partial_{E}$ is a negligible correction of the order of the inverse of the system size. The autocorrelation function is given by

$$
C(\tau)=\left\langle F_{\mathrm{LJ}}(\tau) F_{\mathrm{LJ}}(0)\right\rangle_{E, R}-\left\langle F_{\mathrm{LJ}}\right\rangle_{E, R}^{2}
$$

where $F_{\mathrm{LJ}}$ is the total Lennard-Jones force in the transverse direction,

$$
F_{\mathrm{LJ}}=\frac{1}{2} \sum_{i j} \frac{\partial V_{\mathrm{LJ}}}{\partial R}\left(\mathbf{r}_{i}^{(1)}-\mathbf{r}_{j}^{(2)}\right)
$$

We observe that Eq. (8) satisfies the fluctuationdissipation theorem relating the fluctuating force acting on a system in a bath to the friction kernel in the generalized Langevin equation:

$$
k_{\mathrm{B}} T \zeta(t)=\langle f(t) f(0)\rangle
$$

with $f=F_{\mathrm{LJ}}-\left\langle F_{\mathrm{LJ}}\right\rangle$. Eq. (8) is hence obtained in the Markovian limit where the friction kernel (11) decays on a short time scale such that $\zeta(t)=\zeta_{0} \delta(t)$.

We are now going to show the results of the moleculardynamics simulation. We focus our numerical studies on a specific nanotube namely $(5,0) @(15,0)$ which is commensurate in the classification of Ref. 7]. The inner tube has $N_{1}=60$ atoms and a length of $l_{1}=10.9 \AA$. The outer tube has $N_{2}=240$ atoms and a length of $l_{2}=15.3 \AA$. The relative mass is hence $\mu=576$ amu. The moleculardynamics simulation is straightforward. We first extract the internal nanotube such as the initial distance between both mass centers is $R_{0}=6.0 \AA$. The tubes centers of masses have vanishing initial velocity and angular momentum. We then start the simulation and solve the Newtonian dynamics with a fourth-order Runge-Kutta integration scheme in the microcanonical ensemble. The initial temperature of the system is $300 \mathrm{~K}$, which is introduced by a Maxwell-Boltzmann distribution of the initial velocities and a short early time relaxation. We use a time step of $0.1 \mathrm{fs}$.

First, we can predict the energy to be dissipated by the sliding motion and thus the increase of the temperature of the system. Calculating the difference of energies between the minimum of the total LennardJones potential $V_{\mathrm{LJ}}(R)$ and the initial value $V_{\mathrm{LJ}}\left(R_{0}\right)$ gives us $\Delta E_{s}=0.623 \mathrm{eV}$. Using $\Delta E=3 N k_{B} T$ where $N=N_{1}+N_{2}$ we hence should have an increase in temperature of $\Delta T=8.04 K$.

We observe in Fig. 2] a double exponential decay in the motion. The origin of the double exponential decay holds in the fact that, in the initial configuration, a part of the inner tube is outside the outer tube. In this case, the friction is higher because the inner tube interacts with the edge of the outer tube and bounces during the reentry. It is only after a while that the inner tube oscillates inside the outer tube. We see that the oscillations are damped as expected. We can use a simple model to describe the dynamics when the inner tube remains inside, in which case the potential energy of the relative motion between 

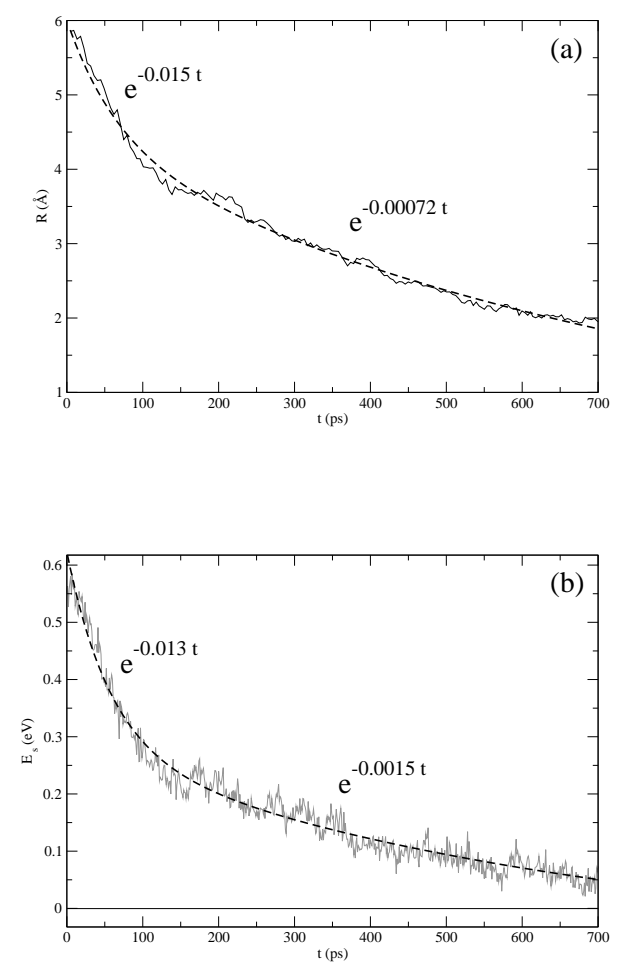

FIG. 2: (a) Evolution of the distance between mass centers represented by the successive maxima at each oscillation. (b) Evolution of the energy of the relative motion also represented by the successive maxima at each oscillation. In this small system, the oscillations have a period of about 10ps. The crossover between the exponentials happens around 120fs when the bouncing of the edges ceases. The inner tube is entirely inside the outer one after about $400 \mathrm{fs}$.

both tubes can be approximated by a harmonic potential. We hence have a Newton equation of the form,

$$
\mu \ddot{R}=-\Gamma \dot{R}-k R
$$

which can be solved to show that the successive maxima in position $R$ and energy $E=\frac{1}{2} \mu \dot{R}^{2}+\frac{1}{2} k R^{2}$ decay as

$$
|R|_{\max } \propto e^{-\Gamma t / 2 \mu} \quad \text { and } \quad E_{\max } \propto e^{-\Gamma t / \mu}
$$

We hence expect to have a ratio of 2 between the damping rates of the maxima in energy and position. By performing fits of the curves in Fig. 2 we get respectively $0.00072 / \mathrm{ps}$ and $0.0015 / \mathrm{ps}$, in good agreement with the expectation. Multiplying by the relative mass $\mu$ we finally get the friction coefficient $\Gamma=0.84 \pm 0.02 \mathrm{amu} / \mathrm{ps}$.

The next step is to calculate the friction coefficient with the autocorrelation-function method. According to Eqs. (8)-(10), the friction coefficient is given by the time integral of the autocorrelation function of the force acting on the slow system with the distance between the centers of mass fixed as a parameter. The calculation of the autocorrelation function is also performed by moleculardynamics simulation except that the distance between
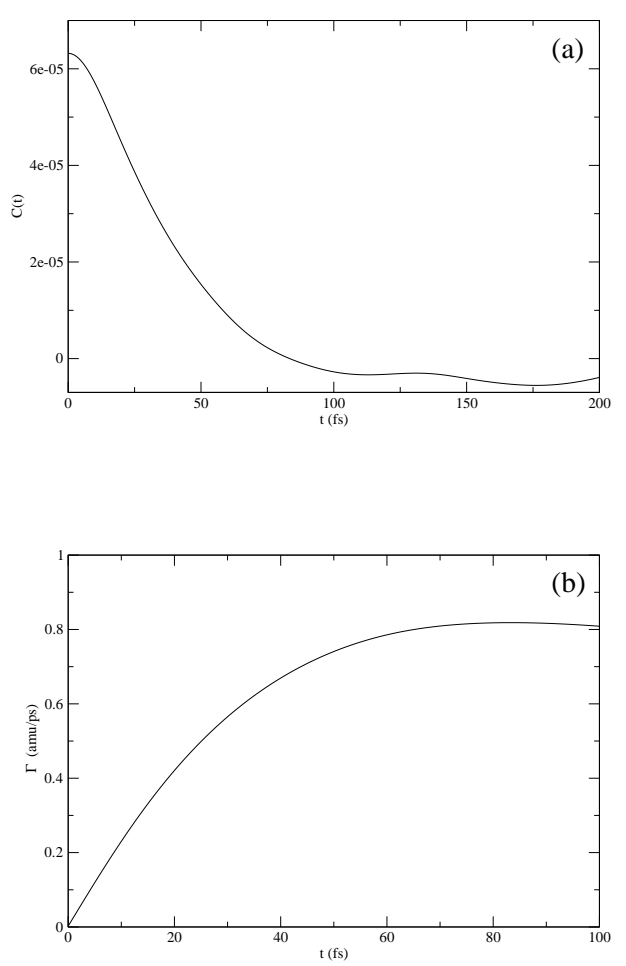

FIG. 3: (a) Autocorrelation function of the intertube force. (b) Time integral of the autocorrelation function (a) numerically converging to the friction coefficient. The small fluctuations seen beyond about $80 \mathrm{fs}$ in the autocorrelation-function (a) and its integral (b) give a negligible contribution and are reduced if the statistics used in the averaging are increased.

the centers of mass is kept constant by compensating at each time step the motion of each atom by the motion of the mass center of their respective nanotube. We suppose that our system is ergodic and mixing (meaning that its autocorrelation functions vanish asymptotically at long times). Since we have nearly one thousand degrees of freedom this should be a good approximation. We can thus use the ergodic theorem and replace the ensemble average in Eq. (9) by a time average.

Figure 3 depicts the results of the numerical calculation of the autocorrelation-function and its time integral giving asymptotically the friction coefficient. With this autocorrelation-function method, we find the value $\Gamma=0.82 \mathrm{amu} / \mathrm{ps}$ for the friction coefficient, in excellent agreement with the value obtained by direct moleculardynamics simulation. The great advantage of the autocorrelation function method is that the calculation is now about 50 times faster. Furthermore the autocorrelation function method is much more precise since it does not involve fitting to get the friction coefficient.

Furthermore, the autocorrelation-function method allows us to obtain a theoretical formula for the friction coefficient. We first notice that the autocorrelation function decays on the time scale of the vibrations of the 
atoms in the carbon nanotubes. This time scale is of the order of the inverse of the Debye frequency $\omega_{\mathrm{D}}$ so that the correlation time should be given approximately by $t_{\mathrm{C}} \approx 2 \pi / \omega_{\mathrm{D}}$. Since the Debye temperature of the nanotube is $\Theta_{\mathrm{D}} \approx 1000 \mathrm{~K}[12$, we hence expect the autocorrelation function to decay to zero over a correlation time approximately equal to $50 \mathrm{fs}$, which is indeed confirmed by Fig. 3] Moreover, the autocorrelation function at time zero is equal to the variance $\Delta F^{2}$ of the force. We can therefore approximate the time integral of the autocorrelation function and obtained the following approximate formula for the friction coefficient:

$$
\Gamma \approx \frac{1}{k_{\mathrm{B}} T} \frac{1}{2} \frac{2 \pi}{\omega_{\mathrm{D}}} \Delta F^{2}
$$

Thanks to this formula, the evaluation of the friction coefficient reduces to the evaluation of the variance of the fluctuating force. On this ground, we now investigate the dependence of the friction coefficient on temperature. We have depicted in Fig. 4 the result of the numerical calculation of the standard deviation of the force. We see

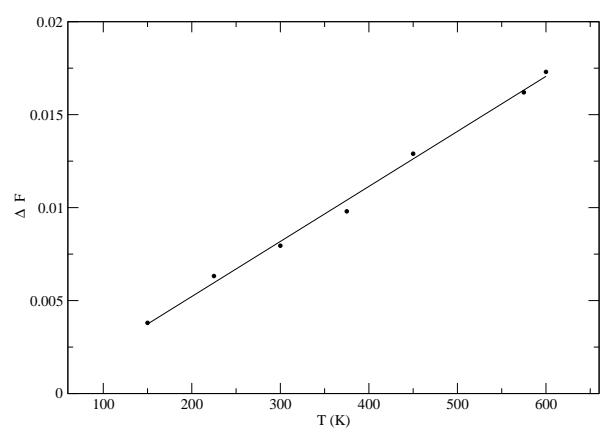

FIG. 4: Standard deviation of the force versus temperature.

that the standard deviation of the force increases linearly with the temperature, hence the friction coefficient also increases linearly with temperature: $\Gamma \sim T$. The fact that friction increases with temperature in our system can be understand by the fact that the dissipation of energy of the slow motion is due to the coupling with the vibrating degrees of freedom. Increasing the temperature results into larger vibrational motion and thus into higher friction. Furthermore, the calculation shows that edge effects give to the small system we here considered, a larger friction than to a system of double length. Then the friction increases in systems from double to longer lengths, the coefficient remaining of the same order of magnitude as in the small system here studied.

In this work, we have developed theoretical and numerical methods to calculate the dynamic friction coefficient in the one-dimensional sliding motion of two concentric carbon nanotubes. We focused our numerical studies on a commensurate double-walled nanotube. We obtained by a molecular-dynamics simulation the energy damping and thus the increase in temperature of the system. The simulation shows the importance of edge effects at early times: a significantly larger damping is due to bouncing effects when the inner nanotube reenters. This effect disappears at long times when the inner tube moves inside the outer tube. The long time behavior is in good agreement with the one-dimensional model we proposed. We have then calculated the friction with the autocorrelation-function method. We observed that the autocorrelation function decreases to zero with a time of the order of $50 \mathrm{fs}$ which is a value in agreement with the experimental value of the Debye temperature. The integral of the autocorrelation function gives the same result as the direct molecular-dynamics simulation with much less calculation time, actually a factor 50, and with better precision since we avoid the use of fitting methods. We can thus conclude that the autocorrelation-function method is very efficient for the calculation of friction coefficients. Moreover, we have obtained a formula which relates the dynamic friction coefficient to the Debye frequency and the standard deviation of the fluctuations of the forces between the nanotubes and determined that the dynamical friction coefficient increases with the temperature.

The Authors thank Pablo Jensen for helpful discussions. This research is financially supported by the National Fund for Scientific Research (FNRS Belgium).
[1] J. Cumings and A. Zettl, Science 289, 602 (2000)

[2] D. W. Brenner, Phys. Rev. B 42, 9458 (1990)

[3] Q. Zheng and Q. Jiang, Phys. Rev. Lett. 88, 045503 (2002)

[4] J. P. Lu and W. Yang, Phys. Rev. B 49, 11421 (1994)

[5] J. P. Lu, Phys. Rev. Lett. 79, 1297 (1997)

[6] S. B. Legoas, V. R. Coluci, S. F. Braga, P. Z. Coura, S. O. Dantas, and D. S. Galvao, Phys. Rev. Lett. 90, 055504 (2003)

[7] A. N. Kolmogorov and V. H. Crespi, Phys. Rev. Lett.
85, $4727(2000)$

[8] C. Jarzynski, Phys. Rev. Lett. 71, 839 (1993)

[9] M. V. Berry and J. M. Robbins, Proc. Roy. Soc. Lond. A. 442, 659 (1993)

[10] E. Ott, Phys. Rev. Lett. 42, 1628 (1979)

[11] H. Goldstein, C. Poole and J. Safko, Classical Mechanics, third edition (Addison Wesley, Reading MA, 2002)

[12] J. Hone, B. Batlogg, Z. Benes, A.T. Johnson and J.E. Fisher, Science 289, 1730 (2000) 